\title{
Is OB/GYN Ultrasound a Science of Uncertainty and an Art of Probability? USA Perspective and Hawaii Experience
}

\author{
${ }^{1}$ Holly Olson, ${ }^{2}$ Ivica Zalud
}

\begin{abstract}
At every level of medical education and training, accrediting bodies have the ability to drive curricular change through the establishment of standards and the accreditation process. This review demonstrates one aspect of documentation of ultrasound training via the evolution of minimum procedures required over the years and documentation of case logs to establish national norms. The utility of the lan Donald Interuniversity Schools of Medical Ultrasound as one means to assist educators by providing access to hands on simulation is highlighted as an efficient mechanism for providers to demonstrate lifelong learning outside of a residency-training program.
\end{abstract}

Keywords: Accreditation, ACGME, Education, Hawaii, Ultrasound

How to cite this article: Olson $\mathrm{H}$, Zalud I. Is OB/GYN Ultrasound a Science of Uncertainty and an Art of Probability? USA Perspective and Hawaii Experience. Donald School J Ultrasound Obstet Gynecol 2018;12(3):153-155.

Source of support: Nil

Conflict of interest: None

Throughout the last several decades, as medical technology has rapidly advanced, it has led to dramatic changes in patient care which have created new challenges in medical education. Often, educators create training programs with incomplete evidence as to how well those programs will achieve their curricular objectives. This is also true in the area of ultrasound education across the continuum of medical training. A recent review in 2017 demonstrated inconsistent evidence of efficacy in educating medical students in clinical ultrasound. ${ }^{1}$ Given that many medical schools in the USA are still struggling to implement curricula to teach the principles and practice of ultrasound, one could look at that review as reason not to invest time and money in that area. This would be very shortsighted and could cause future challenges for the

\footnotetext{
${ }^{1}$ Assistant Professor and Deputy Designated Institutional Official, ${ }^{2}$ Professor and Kosasa Endowed Chair

1,2Department of OB/GYN and Women's Health, John A Burns School of Medicine, Kapiolani Medical Center for Women and Children University of Hawaii, USA

Corresponding Author: Holly Olson, Assistant Professor and Deputy Designated Institutional Official, Department of OB/ GYN and Women's Health, John A Burns School of Medicine, Kapiolani Medical Center for Women and Children, University of Hawaii, Honolulu, Hawaii, USA, e-mail: olsonh@hawaii.edu
}

graduate medical education program directors that expect trainees to acquire a basic level of understanding in the science of ultrasound in preparation for specific training in various clinical specialties. Future research in medical education at the undergraduate and post-graduate level in the area of medical ultrasound should focus on the specific outcomes medical students and residents need prior to graduation, not the competencies required of attending physicians.

Medical education in the USA is organized with separate accrediting bodies that mandate what must be done at each level with the Liaison Committee on Medical Education (LCME) providing accreditation for medical schools, the Accreditation Council for Graduate Medical Education (ACGME) at the residency and fellowship level and the Accreditation Council for Continuing Medical Education (ACCME) at the practicing physician level now commonly referred to as a lifelong learning. Those bodies have enormous influence on the curriculum by the standards and requirements they publish. To use the specialty of obstetrics and gynecology as an example, the program requirements do not specify that residents must be trained in the performance of ultrasound. The actual words ultrasound or sonography are not even present in the document. But the critical program requirements are IV.A.5.a. ${ }^{2}$ that states "Residents must be able to competently perform all medical, diagnostic, and surgical procedures considered essential for the area of practice" and IV.A.5.a). ${ }^{2}$ (a) that further clarifies that residents "must develop and ultimately demonstrate proficiency in obstetric and gynecologic procedures essential for specialty board certification." 2 This requirement mandates that the residency program director be familiar with the ultimate goal of $\mathrm{OB} / \mathrm{GYN}$ residency training: preparing graduates for the care of patients and eligibility for board certification as a measure of their ability to provide the standard of care. The American Board of Obstetrics and Gynecology (ABOG) has included ultrasonography on the list of required procedures for many years. ${ }^{3}$ Both the written and oral exam leading to ABOG physician certification includes questions related to obstetrical and gynecological ultrasound exams and findings. Once board certification is achieved, annual maintenance of certification (MOC) might consist of specific OB/GYN ultrasound competencies. If a physician completed 
subspecialty training (like Maternal Fetal Medicine or Reproductive Endocrinology and Infertility Fellowship as an example), additional ultrasound related competencies need to be clearly demonstrated during ABOG subspecialty written and oral examination as well as subsequent MOC. Emphasisis again on lifelong learning and maintaining critical knowledge and skills to perform and interpret $\mathrm{OB}$ and GYN ultrasound. While ABOG certifies physicians performing $\mathrm{OB} / \mathrm{GYN}$ ultrasound as a part of OB/GYN clinical practice, The American Institute for Ultrasound Medicine accredits OB and GYN clinical practice sites performing ultrasound exams. This is an elaborate peer-review process to ensure ultrasound exams are performed and interpreted in a setting of quality clinical service and patient safety, continuously meeting established national standards.

How program directors arrive at the conclusion that a resident or fellow has achieved competence in the performance of OB/GYN ultrasound varies widely across programs in the USA. Over the years, the Residency Review Committee (RRC) has initiated minimum numbers of procedures that residents needed to achieve prior to graduation. ${ }^{4}$ Importantly, these minimum numbers were not intended to imply that a resident would be competent at that point; but rather they indicate a program that has sufficient volume to ensure residents have opportunities to develop competence. The ACGME strongly cautions against the common practice where trainees will stop logging procedures once they have achieved the minimum number as this does not allow proper determination when programs may have too much volume in a particular area. ${ }^{4}$ A recent article by Gupta et al. in 2015 made a note of changing procedural volumes in obstetrics between 2002 to 2003 and 2012 to 2013. ${ }^{5}$ This article utilized the ACGME case long database but only looked at a few procedures such as vaginal delivery, cesarean delivery, multi-fetal deliveries, amniocentesis, and both forceps and vacuum deliveries. The authors did not include ultrasound in their review. In looking at the data available in the ACGME case log system, the number of ultrasound procedures has changed over the years, and those changes were likely influenced both by changes in practice in addition to changes from the RRC. Looking at the most recent year data are available, 2016 to 2017, the mean number of obstetric ultrasounds was 140 per OB/GYN resident with a standard deviation of 109.1 The minimum number of procedures reported was 27 , and the maximum was 927 , indicating a wide variation in training opportunities. For transvaginal gynecologic ultrasound, the mean was $81.4+/-43.7$ with a minimum of 22 and a maximum of 497.6 These numbers are in contrast to the data from 2006 to 2007 when no obstetric ultrasound data was collected, and the range of vaginal ultrasound procedures varied from a minimum of 0 to a maximum of $1023 .{ }^{7}$ What is perhaps most remarkable is that the RRC never tracked obstetric ultrasound prior to the 2012 to 2013 academic year. This practice most likely related to the history of the committee not tracking the procedures that they felt were so basic to the specialty that everyone would have done them prior to completion of training. Based on the first few years of data recorded, that presumption may have been inaccurate, and it supports the principle that when you count something, training programs pay more attention. This is the mechanism by which accreditation has the power to change educational practices and why case logs form one marker of educational quality.

At the maternal-fetal medicine (MFM) fellowship level, trainees no longer count basic ultrasounds. According to the frequently asked questions regarding the MFM case log entries:

"To reduce the burden of logging, fellows are only required to abnormal ultrasounds: abnormal fetal growth $(<10$ th percentile or $>90$ th percentile), fetal malformations, abnormal placentation, and genetic disorders. Fellows are not required to log ultrasounds with normal findings. However, they may log them for their own purposes. Ultrasounds with normal findings are not being tracked." ${ }^{8}$ Both residents and fellows need to master the following competencies related to OB/GYN ultrasound: patient care, medical knowledge, interpersonal and communication skills, professionalism, practice-based learning, and system-based practice. They should be able to make informed decisions about diagnostic ultrasound modalities based on patient information and preferences, current scientific evidence and clinical judgment. In addition, they should be skilled in performing and/ or interpreting ultrasound exams and counseling and educating patients and their families about ultrasound findings. Trained physicians should use information technology to support patient care decisions and patient education related to ultrasound. Finally, they should work with other healthcare professionals, including those from other disciplines, to provide contemporary patient-focused care.

The essential problem related to counting procedures is that volume alone does not equal competence; there is data in the emergency medicine literature to suggest that proficiency is typically achieved with between 50 to 75 studies when expert reviewers scored videotaped examinations. ${ }^{9}$ This study could serve as a model for other programs to consider mechanisms for determining competence in the performance of ultrasound. This is an area where further educational research must be done 
to provide program directors with the tools they need to ensure the training they are providing is achieving the results they are seeking. Documenting competence is important to our trainees, our programs and the patients they serve.

Lifelong learning is a concept of evidence-based medicine applied to clinical practice in addition to continuous clinical quality improvement and safe environment for our patients. As an example, the Ian Donald Interuniversity School of Medical Ultrasound Hawaii branch has successfully held numerous educational activities starting from $2005 .^{10}$ The primary goals of the learning environment were to effectively and efficiently educate the OB/GYN community in Hawaii on the recent innovations and trends in $\mathrm{OB}$ and GYN ultrasound as well as to provide a solid foundation and resource for those physicians who perform office ultrasounds for their patients. A high-quality ultrasound CME course in Hawaii provided many advantages for local physicians, allied women health care professionals, sonographers, nurses, geneticists and visiting professionals attending the conference. Our educational activities offered the Hawaii community the opportunity to participate in a large scale, specialized learning environment in ultrasound without having to shoulder the burden of expensive travel arrangements and closing their practices for an extended period. It also gave visiting ultrasound professionals a glimpse into clinical environment experienced by our unique patient population in Hawaii. The authors hopes that the Hawaii courses will continue to provide high-quality lifelong learning ultrasound experience and advance clinical care. The 7th Bi-annual educational and scientific Ian Donald Interuniversity School of Medical Ultrasound Hawaii Branch CME approved meeting is planned for December 14 to 15, 2018 in Honolulu with internationally highly recognized faculty: Drs. Kurjak, Merz, Chervenak, Lookwood, and Schafer, and Librizzi.

Ultrasound is continuously enhancing and improving the practice of everyday OB/GYN care. As ultrasound technology becomes more sophisticated, the imaging professional requires lifelong learning to maintain proficiency. The medical educators' responsibility is to ensure that learners understand the conscientious use of new technology to improve and maintain the health of our patients. Delivery of efficient and effective instructions requires respect for adult learning theory while ensuring that the instruction is untainted by commercial bias. ${ }^{11}$ Hands-on learning via simulation can be very effective, and ultrasound simulations should be incorporated into learning events, whenever possible. The role of medical student and residents/fellows education and board certification followed by Maintenance of Certification and Maintenance of Licensure, in the lifelong learning realm, will continue to be further defined. Our aim is OB/GYN ultrasound as a science of certainty and less as an art of probability. Ian Donald Interuniversity Schools of Medical Ultrasound are working enthusiastically and diligently on this worldwide.

\section{REFERENCES}

1. Feilchenfeld Z, Dornan T, Whitehead C, Kuper A. Ultrasound in undergraduate medical education: a systematic and critical review. Medical education. 2017 Apr;51(4):366-378.

2. Accreditation Council for Graduate Medical Education (USA). Obstetrics and Gynecology. 2017 [cited 2018 June 2].Available from: https://acgme.org/Specialties/ProgramRequirements-and-FAQs-and-Applications / pfcatid/12/ Obstetrics and Gynecology.

3. American Board of Obstetrics and Gynecology. 2017 [cited 2018 Oct 12]. Available from: https://www.abog.org/docs/ default-source/abog-bulletins/2018-certifying-examinationin-obstetrics-and-gynecology.pdf?sfvrsn=20ad3a0_2.

4. Accreditation Council for Graduate Medical Education (USA). Obstetrics and Gynecology [Internet]. 2017 [cited 2018 June 2]. Available from: https://acgme.org/Portals/0/PFAssets/ ProgramResources/220_Ob_Gyn_Minimum_Numbers_ Announcement.pdf?ver=2018-06-25-104354-993.

5. Gupta N, Dragovic K, Trester R, Blankstein J. The Changing Scenario of Obstetrics and Gynecology ResidencyTraining. J Grad Med Educ. 2015;7(3):401-406.

6. Accreditation Council for Graduate Medical Education (USA). Case Logs Statistical Reports. 2017 [cited 2018 June 2]. Available from: http://www.acgme.org/Portals/0/ PDFs/220_National_Report_Program_Version_2016-2017. pdf.

7. Accreditation Council for Graduate Medical Education (USA). Case Logs Statistical Reports [Internet]. 2007 [cited 2018 June 2]. Available from: http:/ /www.acgme.org/Portals/0/PDFs/ ObGynNationalData0607. pdf.

8. Accreditation Council for Graduate Medical Education (USA). Obstetrics and Gynecology [Internet]. 2018 [cited 2018 June 2]. Available from: http:/ / www.acgme.org/Portals/0/ PFAssets/ProgramResources/MFM_Case_Log_Instructions. pdf?ver=2018-01-16-123634-450.

9. J. BD, Bruce B, J. GR. Learning Curves in Emergency Ultrasound Education. Acad Emerg Medicine. 2015;22(5):574-582.

10. Tsai PJ S, Wong S, Zalud I: Ultrasound education in Obstetrics and Gynecology: Hawai'i Experience. Hawaii J Med Pub Health 2013; 72:172-177.

11. Aeby TC, Wong S, Zalud I: Current trends in obstetrics and gynecology ultrasound continuing medical education. Donald School J Ultrasound Obstet Gynecol 2014;8(1):60-64. 